\title{
Non-hyperbolic discounting and dynamic preference reversal
}

\author{
Shou Chen ${ }^{1} \cdot$ Richard Fu $^{2} \cdot$ Lei Wedge $^{3} \cdot$ Ziran Zou $^{1}$
}

Published online: 9 January 2019

(c) The Author(s) 2019

\begin{abstract}
In this paper, we present a time-varying and non-stationary but non-hyperbolic discount function that explains dynamic preference reversal. The new discount function emerges from an analysis of intertemporal consumption and savings choices with mortality risk and an altruistic factor. Our analysis shows that the process of updating survival information may also account for dynamic preference reversal.
\end{abstract}

Keywords Dynamic preference reversal · Non-hyperbolic discounting · Time varying · Updating information

\section{Introduction}

Decision-making processes frequently involve updating information. A decision maker (henceforth DM) may change her intertemporal preferences over time as she updates uncertain information in the process, which may lead to apparently timeinconsistent behaviors. For example, abundant anecdotal evidence shows that people tend to reverse their original retirement plans when approaching the planned retirement age. They update uncertain survival information over time. Those in good health who expect to live longer are apt to continue working to accumulate more retirement pension in the future, while those in poor health are apt to stop working to receive less but earlier retirement pension.

$凶$ Ziran Zou

zouziran@hnu.edu.cn

1 Business School of Hunan University, Changsha 410082, Hunan, China

2 Collat School of Business, University of Alabama at Birmingham, Birmingham, AL 35294, USA

3 Muma College of Business, University of South Florida, Tampa, FL 33620, USA 
Time-inconsistent behavior is also termed dynamic preference reversal, ${ }^{1}$ which is well documented in academic research: the DM prefers a larger delayed reward to a smaller immediate reward but reverses her preferred choice in the future as a future time draws nearer. ${ }^{2}$ Under the assumption of time invariance, dynamic preference reversal is equivalent to static preference reversal (Frederick et al. 2002): the phenomenon whereby a DM prefers a smaller immediate reward over a larger delayed one but reverses her preferences when these two alternatives are equally shifted to the future. ${ }^{3}$ In short, preference reversals resulting from a change in decision time ${ }^{4}$ are termed "dynamic", and a DM exhibiting dynamic preference reversal has timeinconsistent preferences. In contrast, reversals from an equal time shift from two future alternatives at the same decision time are termed "static", and a DM exhibiting statistic preference reversal has non-stationary preferences. Numerous studies implicitly make the assumption of time invariance without distinguishing static from dynamic preference reversal, and they simply refer to these terms together as preference reversals or time-inconsistent choices. Furthermore, with the assumption of time invariance, non-stationary (stationary) preferences are equivalent to time-inconsistent (time-consistent) preferences. ${ }^{5}$ However, in general, non-stationarity (stationarity) is not always equivalent to time inconsistency (time consistency). For example, Read (2004) demonstrates that a DM shows dynamic preference reversals but no static preference reversals, which means that the DM has stationary but time-inconsistent preferences.

In fact, the stationarity, time consistency and time invariance of preferences are similar yet distinctively different concepts. Stationarity means that at any fixed decision time, a preference over two future rewards depends only on the time distance and reward difference between these two alternatives. For example, Keren and Roelofsma (1995) show that when making decisions at the current time, $82 \%$ of their subjects prefer to receive $\$ 110$ after 30 weeks rather than $\$ 100$ after 26 weeks, and $37 \%$ of their subjects prefer to receive $\$ 110$ after 4 weeks rather than $\$ 100$ immediately. Those subjects are said to have stationary preferences because their preferences at a fixed decision time over two pairs of future alternatives with the same distance and reward difference do not change. However, $45 \%$ of their subjects prefer $\$ 100$ immediately rather than $\$ 110$ after 4 weeks. These subjects are said to have nonstationary preferences because their preferences at a fixed decision time over two pairs of future alternatives are affected by the difference between the reward times and

\footnotetext{
1 Many studies use the term "dynamic preference reversals" interchangeably with "time-inconsistent choices" (see, for example, Hoch and Loewenstein 1991). However, Sayman and Öncüler (2009) find evidence of reversal dynamic preference reversals, which are actually time-inconsistent choices.

2 Strotz (1955) and O'Donoghue and Rabin (1999) provide the examples of dynamic preference reversal in real life, while Halevy (2015) shows evidence of this through experiments.

3 Static preference reversal is also referred to as non-stationary behavior and synchronic preference reversal. Dynamic preference reversal is referred to as diachronic preference reversal in Frederick et al. (2002).

4 Tversky and Kahneman (1991) first show that changes in a decision time often lead to reversals of preferences. In this paper, the decision time can be either the present time or a future time and may also coincide with the decision time.

5 Attema et al. (2010) also use the term "homogenous time" to describe time invariance. They note that under the assumption of homogeneous time, the condition of non-stationary preferences is equivalent to the condition of time-inconsistent preferences.
} 
the decision times. Time consistency means that a preference between two fixed future rewards remains the same at any decision time as long as the time and reward difference between these two alternatives remains the same. Take as an example the subjects who currently prefer to receive $\$ 110$ after 30 weeks rather than $\$ 100$ after 26 weeks. As time goes by, say, in 26 weeks, if they still prefer $\$ 110$ in 4 weeks to $\$ 100$ immediately, they are said to have time-consistent preferences. In everyday life, however, we see many examples of time inconsistency: people often deviate from their original savings plans for retirement by consuming more than initially planned. Time invariance means that a preference for any future two rewards with the same time distance and reward difference does not change as long as the time distances between the decision time and rewards remain the same. For example, for an experiment conducted on Jan 1, 2018, a subject prefers to receive $\$ 110$ after 30 weeks rather than $\$ 100$ after 26 weeks. When this experiment is repeated on Jan 1, 2019, the subject's preference is considered time invariant if her decision does not change.

Using the axiomatic method, Halevy (2015) is the first to show that the presence of any two of the three properties (time invariance, time consistency and stationarity) implies the presence of the third. This theoretical result means that under the assumption of time invariance, the DM's intertemporal preferences are time consistent if and only if these preferences are stationary. The concept of stationarity is premised on a fixed decision time; i.e., the time that the DM has to decide which alternative is more preferable does not change. In contrast, by its very definition, time consistency involves a shift in decision time. In other words, time consistency characterizes whether the DM's preferences over future alternatives remain unchanged (time consistent) or not (time inconsistent) as the decision time goes by while the future delivery time of the two rewards is fixed. Naturally, time invariance provides a crucial link that connects these two distinct notions. The functional form of a timeinvariant preference is not dependent on calendar time. In other words, time-invariant preferences over two future rewards depend only on the time distance and reward difference between two future alternatives and the time distance between the decision time and the reward time, regardless of whether the decision time changes. Consequently, stationarity and time consistency are equivalent. Likewise, non-stationarity is equivalent to time inconsistency when a preference is time invariant. Therefore, under the assumption of time invariance, dynamic preference reversal is equivalent to static preference reversal.

Economists generally believe that the present bias, which characterizes the DM's non-stationary preferences, accounts for static preference reversal. Recent economic literature focuses on time-invariant hyperbolic discounting as a form of the present bias to explain both static and dynamic preference reversals. ${ }^{6}$ Since hyperbolic discounting explains preference reversals and fits experimental data well while exponential

\footnotetext{
6 A large body of literature (e.g., Phelps and Pollak 1968; Loewenstein and Prelec 1992; Scholten and Read 2006; Noor 2011; Harris and Laibson 2013; Pan et al. 2015) shows that non-exponential discount functions with the time-invariant assumption can explain preference reversals. Another body of literature implicitly assumes that the DM has time-varying preferences, and this research uses hyperbolic discount functions to explain dynamic preference reversals, e.g., Azfar (1999) and Dasgupta and Maskin (2005).
} 
discounting does not, preference reversals, especially the existence of dynamic ones, are regarded as evidence of hyperbolic discounting (Frederick et al. 2002). ${ }^{7}$

Halevy's (2015) experiments show that at least half of subjects are time consistent. However, only three-quarters of them exhibit stationary choice behavior, while approximately half of subjects with time-inconsistent choices have stationary preferences. These results imply that both time-varying and non-stationary behavior contribute to time-inconsistent behaviors. In fact, a person may be time inconsistent because of non-stationary preferences, time-varying preferences or both. This notion indicates that there may exist other explanations for time inconsistency besides the present bias, which describes non-stationarity. Halevy (2015) does not investigate the underlying causes of time-varying and non-stationary preferences. Motivated by Halevy (2015), we delve into a theoretical analysis of potential underlying causes of dynamic preference reversal. ${ }^{8}$ We show that the process of updating information, which renders the DM's preferences time varying and non-stationary, ultimately leads to dynamic preference reversal. ${ }^{9}$ Consequently, the process of updating information may be another cause of dynamic preference reversal. To some extent, it is reasonable to assume time invariance for inter-temporal preferences, particularly in problems with a short time horizon. With the assumption of time invariance, non-stationarity is equivalent to time inconsistency. Therefore, the present bias explains both dynamic and static preference reversals. However, in general, the assumption of time invariance is untenable, as shown in this study.

In our analysis, we first characterize the three inter-related notions of time invariance, stationarity and time consistency by applying the concept of the marginal rate of substitution, which can be defined at any decision time occurring at or before the delivery of future rewards. We use the marginal rate of substitution to identify discounting behavior. The flexibility in choosing the decision time enables us to analyze how the process of updating information affects the DM's preferences in our framework. We establish a sufficient condition for dynamic preference reversal characterized by the marginal rate of substitution. Next, following the frameworks developed by Phelps and Pollak (1968), Halevy (2005) and Ekeland and Pirvu (2008), we incorporate mortality risk and an altruistic factor into discount modeling. ${ }^{10}$ We show that the resulting discount function is time varying and non-stationary and is similar to, yet distinct from, a hyperbolic discount function. The DM characterized by this discount function engages in dynamic preference reversal. More specifically, our results show that the process of updating information may be one of the underlying causes of dynamic preference reversal.

\footnotetext{
7 In fact, the example provided by Read (2004) shows that an individual has stationary preferences but exhibits dynamic preference reversal. Furthermore, an individual has time-consistent preferences but engages in static preference reversal in Halevy (2005) model.

8 We focus on only one type of time-inconsistent choice, i.e., dynamic preference reversal. We do not consider other types of time-inconsistent choices such as those described in Sayman and Öncüler (2009).

9 In fact, the DM considered in our model has time-varying preferences and engages in dynamic preference reversal because of her updating survival information over time. However, the DM considered in the model developed by Dasgupta and Maskin (2005) has time-varying preferences and engages in dynamic preference reversal by updating earlier realization information on rewards over time.

10 The altruistic factor is a parameter that differentiates utility derived from the DM's own consumption and bequest to her heirs, as first proposed by Phelps and Pollak (1968).
} 
Our study makes several contributions to the literature. We follow the frameworks developed by Sozou (1998), Azfar (1999) and Halevy (2005) in modeling discounting, but we apply a different approach to explain dynamic preference reversal. We consider mortality risks and utility from bequests in our discounting model. Hence, we obtain results that are markedly different from those of Sozou (1998) and Halevy (2005). Those studies assume no utility derived from bequests and consequently predict no dynamic preference reversal behavior. ${ }^{11}$ In our model, the discount value increases as the DM updates her survival information over time, which has a stronger effect on an earlier reward than on a later reward. As a result, the marginal rate of substitution decreases with the passage of decision time, leading to dynamic preference reversal behavior. ${ }^{12}$ Second, we present a non-hyperbolic and time-varying discount function that explains dynamic preference reversal. Hence, it is premature to interpret the existence of dynamic preference reversal behavior as definitive evidence of hyperbolic discounting. ${ }^{13}$ Finally, our analysis, in addition to that of Dasgupta and Maskin (2005), provides a theoretical underpinning for the possibility that the process of updating information may account for time-varying preferences, which lead to dynamic preference reversal behavior. Halevy (2015) does not investigate the underlying causes of time-varying and non-stationary preferences, and our results offer a theoretical explanation for his experimental results.

\section{Preference reversals and discounting}

It was well established by Halevy (2015) that the presence of any two of the three properties of time invariance, time consistency and stationarity implies the existence of the third. This result means that under the assumption of time invariance, the DM's intertemporal preferences are time inconsistent if and only if the preferences are nonstationary.

Preference reversals have been widely studied in economics research. Under the assumption of time invariance, the DM exhibits static preference reversal if and only if she shows dynamic preference reversal (Frederick et al. 2002). Therefore, presentbiased preferences that explain static preference reversal can also explain dynamic preference reversal under the assumption of time invariance. ${ }^{14}$ Figure 1 illustrates the relationship among present-biased preferences, dynamic preference reversal, static preference reversal and time invariance.

Present-biased preferences are a model of hyperbolic discounting, which is a natural way to represent how the DM responds to time delays (O'Donoghue and Rabin

\footnotetext{
11 Readers are referred to Dasgupta and Maskin (2005) for a detailed discussion of why Sozou's model (1998) does not explain dynamic preference reversal.

12 Our results are distinct from those of Halevy (2005). In his paper, although the discount function is similarly time varying, the DM's choice is time consistent, as the discount value increases proportionally for the two different time alternatives during the updating of her survival probabilities over time.

13 Our results are different from those of Dasgupta and Maskin (2005), who find that discounting behavior is hyperbolic, while our discount function is non-hyperbolic. In their study, the DM's dynamic preference reversal behavior results from updating the information from the early realization of rewards.

14 The phenomenon of static preference reversal is referred to as present bias in Halevy (2015).
} 


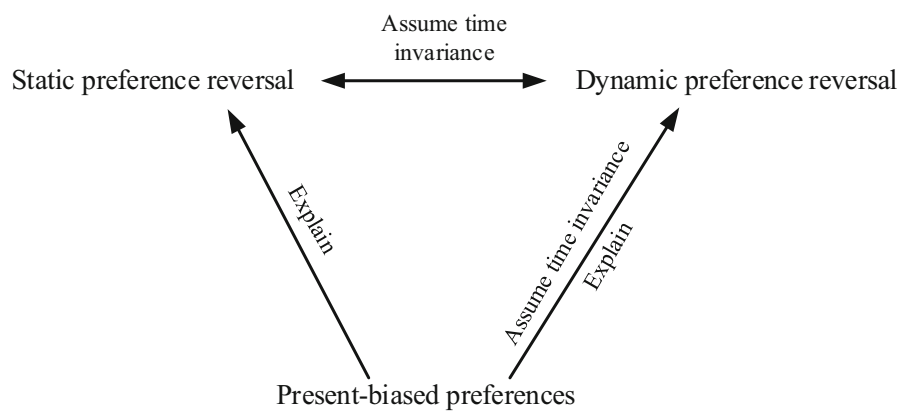

Fig. 1 The relationship between static and dynamic preference reversal

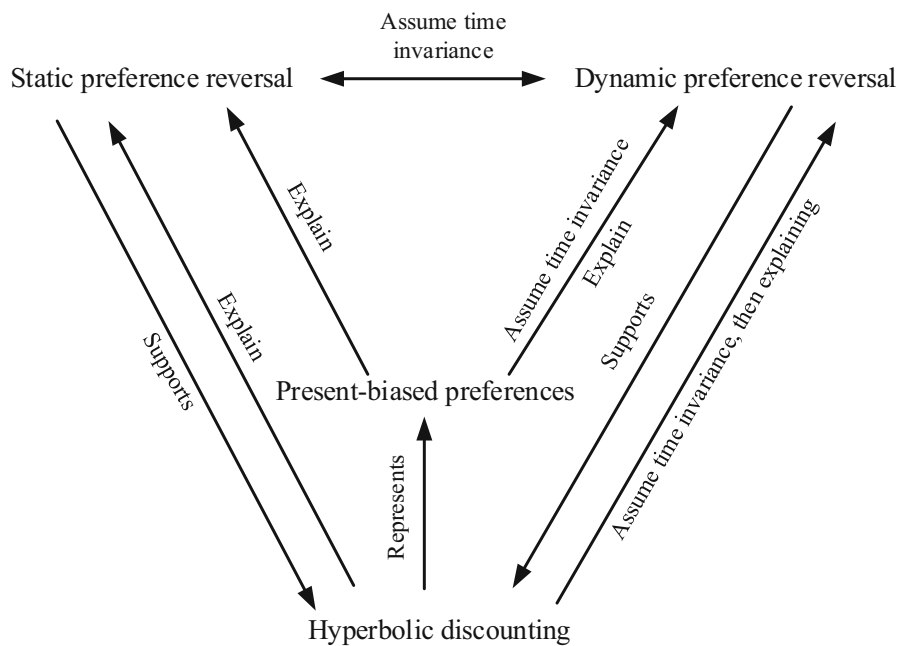

Fig. 2 The relationship between static, dynamic preference reversal and hyperbolic discounting

2015). ${ }^{15}$ The most popular models are the quasi-hyperbolic discounting (Phelps and Pollak 1968) and hyperbolic discounting functions proposed by Harvey (1986), Mazur (1987) and Loewenstein and Prelec (1992). All of these implicitly assume time invariance and explain both static and dynamic preference reversals from the perspective of discounting. The existence of dynamic preference reversal is regarded as evidence of hyperbolic discounting. ${ }^{16}$ Figure 2 illustrates the relationship among hyperbolic discounting, present-biased preferences, time invariance, dynamic preference reversal and static preference reversal.

\footnotetext{
15 Hyperbolic discounting is also referred to as diminishing impatience, and the present bias is a particular case of diminishing impatience (Pennesi 2017). A huge body of literature addresses a wide range of issues in economics based on present bias, which includes Grenadier and Wang (2007), Zou et al (2014) and Chen et al (2014), among others.

16 In the model developed by Dasgupta and Maskin (2005), discounting behavior is hyperbolic and time varying, but this model can explain dynamic preference reversal.
} 
The experimental results presented in Halevy (2015) show that the DM has timevarying preferences and that time-inconsistent behavior can occur as a result of nonstationary preferences, time-varying preferences or both. Therefore, in addition to the present bias, there may exist other causes that account for dynamic preference reversals. We will show that the process of information updating is an underlying cause of dynamic preference reversal behavior.

The decision time plays a very important role in revealing the processes of information updating as an underlying cause of dynamic preference reversal behavior. We next define preferences and incorporate the decision time into preferences to show that the DM's preferences may change as the decision time changes.

Let $[0, X]$ denote the set of outcomes, and let $[0, T]$ denote the set of time at which an outcome occurs, where $X>0$ and $T>0$. A typical element of $[0, T] \times[0, X]$ is $\left(t^{\prime}+\right.$ $\Delta, x)$, which represents the receipt of outcome $x$ at time $t^{\prime}+\Delta$. Let $\succsim_{t}$ represent the DM's preferences over temporal payments at time $t$. We also use $\left\{\succsim_{t}\right\}_{t=0}^{\infty}$ to denote the dynamic preference structure. The DM's preferences $\succsim_{t}$ over timed outcomes $\left(t^{\prime}\right.$ $+\Delta, x)$ can be represented by discounted utility $\mathrm{DU}_{t}$ :

$$
\mathrm{DU}_{t}\left(t^{\prime}+\Delta, x\right)=D\left(t, t^{\prime}+\Delta\right) u(x) \text {. }
$$

That is, $\left(t^{\prime}+\Delta_{1}, x\right) \succsim_{t}\left(t^{\prime}+\Delta_{2}, y\right)$ if and only if $\mathrm{DU}_{t}\left(t^{\prime}+\Delta_{1}, x\right) \geq \operatorname{DU}_{t}\left(t^{\prime}+\Delta_{2}, y\right)$. $\mathrm{DU}_{t}$ is the discounted utility at decision time $t$, and $u(x)$ is the utility of payment $x$. $D\left(t, t^{\prime}+\Delta\right)$ is the discount function that denotes the value of one unit of utility at time $t$ to be received at time $t^{\prime}+\Delta .^{17}$

The discounted utility at $t$ over the timed outcome $\left(t^{\prime}, x\right)$ depends on the discount function $D\left(t, t^{\prime}\right)$ and on the utility of payment $u(x)$. In the case of dynamic preference reversals, which result from a shift in the decision time, i.e., $\left(t^{\prime}, x\right) \prec_{t}\left(t^{\prime}+\tau, y\right)$ and $\left(t^{\prime}, x\right) \succ_{s}\left(t^{\prime}+\tau, y\right)$, where $t<s<t^{\prime}, \tau>0$ and $x<y$; timed outcomes $x$ and $y$ are fixed at fixed times $t^{\prime}$ and $t^{\prime}+\tau$; and the ratio between the discounted utility at $t$ over timed outcomes $\left(t^{\prime}, x\right)$ and $\left(t^{\prime}+\tau, y\right), \frac{D\left(t, t^{\prime}+\tau\right)}{D\left(t, t^{\prime}\right)} \frac{u(y)}{u(x)}$, depends only on the discount factors $D\left(t, t^{\prime}\right)$ and $D\left(t, t^{\prime}+\tau\right)$ as the decision time $t$ changes. The discount factors $D\left(t, t^{\prime}\right)$ and $D\left(t, t^{\prime}+\tau\right)$ for the two timed rewards $x$ and $y$ increase as the decision time $t$ changes. However, the discount factor for the earlier reward is not proportional to the later reward. Therefore, the marginal rate of substitution, $\frac{D\left(t, t^{\prime}+\tau\right)}{D\left(t, t^{\prime}\right)}$, changes with the decision time, which may lead to dynamic preference reversal. Following Halevy (2005) and Frederick et al. (2002), we refer to this marginal rate of substitution as time discounting or as the value at $t^{\prime}$ of receiving a unit utility at $t^{\prime}+\tau$ from the decision time of $t$. This can be used to determine whether the corresponding discounting behavior is hyperbolic.

We next use the marginal rate of substitution to show that the process of updating information may be one underlying cause of dynamic preference reversal and to determine whether the corresponding discounting behavior is hyperbolic. We define the marginal rate of substitution at $t^{\prime}+\Delta_{1}$ for utility at $t^{\prime}+\Delta_{2}$ from the decision time $t$ as follows:

\footnotetext{
17 We apply the axiomatic framework for preferences shown in Fishburn and Rubinstein (1982) to characterize $\mathrm{DU}_{t}$.
} 


$$
\operatorname{MRS}_{t}\left(t^{\prime}+\Delta_{1}, t^{\prime}+\Delta_{2}\right)=\frac{D\left(t, t^{\prime}+\Delta_{2}\right)}{\left.D\left(t, t^{\prime}+\Delta_{1}\right)\right)}, \quad \Delta_{2}>\Delta_{1}
$$

Following the definitions of time invariance, time consistency and stationarity provided in Halevy (2015) and the preferences represented in Eq. (1), we characterize time invariance, time consistency and stationarity as the marginal rate of substitution as follows:

Proposition 1 For every $\Delta_{1}, \Delta_{2}>0$ and $t, t^{\prime}>0$, preference $\left\{\succsim_{t}\right\}_{t=0}^{\infty}$

(1) is time invariant if and only if the marginal rate of substitution of any future two rewards with the same time distance and reward difference does not change as long as the time distance between the decision time and rewards remains the same, i.e,

$$
\operatorname{MRS}_{t}\left(t+\Delta_{1}, t+\Delta_{2}\right)=\operatorname{MRS}_{t^{\prime}}\left(t^{\prime}+\Delta_{1}, t^{\prime}+\Delta_{2}\right)
$$

(2) is stationary if and only if at any fixed decision time, the marginal rate of substitution over two future rewards depends only on the time distance and reward difference between these two alternatives, i.e,

$$
\operatorname{MRS}_{t}\left(t+\Delta_{1}, t+\Delta_{2}\right)=\operatorname{MRS}_{t}\left(t^{\prime}+\Delta_{1}, t^{\prime}+\Delta_{2}\right)
$$

(3) is time consistent if and only if the marginal rate of substitution over two fixed future rewards remains the same at any decision time as long as the time distance and reward difference between these two alternatives remain the same, i.e,

$$
\operatorname{MRS}_{t}\left(t+\Delta_{1}, t+\Delta_{2}\right)=\operatorname{MRS}_{t^{\prime}}\left(t+\Delta_{1}, t+\Delta_{2}\right) .
$$

\section{Proof See Appendix 1.}

From Proposition 1, stationarity precludes static preference reversal, and time consistency precludes dynamic preference reversal. Furthermore, it is evident that under the assumption of time invariance, the DM exhibits static preference reversal if and only if she engages in dynamic preference reversal. ${ }^{18}$ Although a variety of hypotheses have been proposed for the origins of these behaviors, ${ }^{19}$ most economists agree that static preference reversal is typically attributed to present bias. ${ }^{20}$ Hyperbolic discounting is usually defined as (for a fixed decision time) an increase in the marginal rate of substitution with two alternatives shifting equally into the future (see Read

\footnotetext{
18 Attema et al. (2010) use the term "homogenous time" to describe the notion of time invariance. They show that under the assumption of homogeneous time, non-stationarity in preferences is equivalent to time inconsistency.

19 For example, Loewenstein and O'Donoghue (2002) point out that visceral influences and similarities in current settings among historical consumption sites may account for static preference reversal.

20 Gul and Pesendorfer (2001) propose an alternative approach to model time-consistent preferences, suggesting that temptation but not preference change might be the cause of time-inconsistent behavior. Miao (2008) adopts the Gul-Pesendorfer approach to solve the problem of the optimal option exercise using the dynamic programming technique.
} 
2001; Read and Roelofsma 2003; Scholten and Read 2006; Noor (2009); Kinari et al. 2009; Halevy 2005), i.e., $\frac{d \mathrm{MRS}_{t}\left(s+\Delta_{1}, s+\Delta_{2}\right)}{d s}>0 .{ }^{21}$ Time-invariant hyperbolic discount functions can explain both static and dynamic preference reversals.

The assumption of time invariance is untenable in real life because of the presence of a variety of risks. ${ }^{22}$ Such risks may be due to an uncertain lifetime that may prevent the DM from realizing contracted utility. When an uncertain lifetime is considered in the decision-making process, the DM's discounting behavior is dependent on the calendar time because of the DM's non-constant hazard rate of death. Hence, static preference reversal may not be equivalent to dynamic preference reversal, and there may exist other causes to account for dynamic preference reversal. We establish a sufficient condition for dynamic preference reversal as follows:

Proposition 2 The DM exhibits dynamic preference reversal if the marginal rate of substitution $\mathrm{MRS}_{t}\left(t^{\prime}+\Delta_{1}, t^{\prime}+\Delta_{2}\right)$ decreases as the decision time tincreases.

Proof See Appendix 2.

Proposition 2 shows that if the marginal rate of substitution decreases with the decision time, the DM will exhibit dynamic preference reversal. In such cases, the DM's preferences may be time varying or non-stationary or both. ${ }^{23}$ Notably, a decreasing marginal rate of substitution with decision time is only a sufficient condition for dynamic preference reversal. Other conditions may also explain dynamic preference reversal or other time-inconsistent choices. ${ }^{24}$

In the next section, we investigate possible underlying causes of dynamic preference reversal by constructing a discounting model incorporating mortality risk, bequest motivations and survival information updating. We show that the discount function derived from this realistic yet mathematically tractable model is time varying, nonstationary and non-hyperbolic and accounts for dynamic preference reversal well.

\section{Non-hyperbolic discounting and dynamic preference reversal}

In this section, we construct a non-hyperbolic discount function that is time varying with the process of updating information but that explains dynamic preference reversal behavior well. We derive this new discount function from an analysis of a fundamental problem of lifetime consumption with mortality risk. As argued in Yaari (1965) and recently in Sozou (1998), Dasgupta and Maskin (2005) and Halevy (2005), discounting originates from the possible inability of the DM to enjoy future payoffs. We follow

\footnotetext{
21 Using the marginal rate of substitution to define hyperbolic discounting can prevent the influence of interval effects, as shown by Read (2001).

22 Halevy (2015) notes that the risks and demands of liquidity may lead the DM to have time-varying preferences.

23 The discount function used in Dasgupta and Maskin (2005) to explain dynamic preference reversals is time varying and non-stationary, and the corresponding marginal rate of substitution decreases with the decision time.

24 For example, when the marginal rate of substitution increases with the decision time, the DM will engage in reversal dynamic preference reversal, as found by Sayman and Öncüler (2009).
} 
Yaari's (1965) approach by considering mortality risk in our framework. The discount function thus derived encompasses the key elements of pure time preferences of exponential form $\left(\mathrm{e}^{-\rho t}\right)$, an "altruistic factor" $(\beta)$, and survival probability (the Weilbull distribution, $\mathrm{e}^{-\lambda t^{\gamma}}$ ). Our approach is analogous to that of Halevy (2005) in that the primary source of discounting is the mortality risk of the DM, which makes it possible that the DM will not enjoy the fruits of future income streams. Through the process, the DM constantly updates her survival probability, causing her preferences to change over time and resulting in her engagement in dynamic preference reversal behavior. $^{25}$ Our conclusion, together with those of Dasgupta and Maskin (2005), provides a theoretical explanation for how the process of updating uncertain information may be another source of dynamic preference reversal, consistent with the experimental results of Halevy (2005). ${ }^{26}$

Consider an individual who faces mortality risks when making intertemporal decisions on consumption and savings. The probability that she will be alive at time $t$ is given by the survival probability function:

$$
\Omega(t)=e^{-\lambda t^{\gamma}}
$$

where $\lambda>0$ and $\gamma>0$. Life expectancy is the reciprocal of $\lambda$. Parameter $\gamma$ is the monotonicity of the hazard rate of death. Cases $0<\gamma<1, \gamma=1$ and $\gamma>1$ correspond to the burn-in, adult and wear-out periods, respectively.

The probability that the DM will be alive at time $s$ conditional on being alive at time $t$ is:

$$
\Omega_{t}(s)=\frac{\Omega(s)}{\Omega(t)}=\mathrm{e}^{-\lambda\left(s^{\gamma}-t^{\gamma}\right)} .
$$

The hazard rate of death is:

$$
\pi(s)=\frac{-\Omega^{\prime}(s)}{\Omega(s)}=\lambda \gamma s^{\gamma-1} .
$$

The uncertain lifetime prevents the DM from realizing the entire stream of future contracted utility $u(c(s))$, which may be inherited by her heirs after her death. Unlike in previous studies (Yaari 1965; Sozou 1998; Halevy 2005) that assume no utility from bequests, we maintain that both the DM's own consumption in her lifetime and her bequests to heirs have utility for her. ${ }^{27}$ Moreover, utility derived from one's own consumption and bequests differs due to, for example, inheritance tax or self-interest

\footnotetext{
25 Dasgupta and Maskin (2005) consider the uncertainty of disappearing future payoffs and their early realizations. The resulting discount function is hyperbolic and time varying and is consistent with dynamic preference reversal behavior resulting from the DM updating information received early on.

26 Based on experimental results, Halevy (2015) challenges the view that present-biased preferences are the main source of time-inconsistent choices.

27 Roughly $80 \%$ of the capital held by households is inherited (Kotlikoff and Summers, 1981). Kuehlwein (1993) shows that elderly households leave bequests as high as their own consumption. De Nardi (2004) shows that the bequest motive is quantitatively important in explaining the wealth accumulation behavior of the richest people in Sweden.
} 
priorities. Following Phelps and Pollak (1968), we use the "altruistic" factor $\beta$, where $0<\beta<1,{ }^{28}$ to differentiate the utility derived from the DM's own consumption from that derived from bequests to her heirs. The higher $\beta$ is, the more the DM values the expected utility enjoyed by her heirs, thus facilitating a more "altruistic" attitude. We focus on the case of an altruistic DM with a non-constant hazard rate of death, i.e., $\gamma$ $>1$, which leads the DM to have time-varying and non-stationary preferences. ${ }^{29}$

We analyze the present value (at time $t$ ) of the expected utility $u(c(s)$ ) of future consumption at $s$. At time $s$, if she is alive, the expected present value of future utility $u(c(s))$ at $t$ is:

$$
\Omega_{t}(s) \mathrm{e}^{-\rho(s-t)} u(c(s))
$$

If she dies at time $s_{1}, s_{1} \in(t, s)$, the utility of her future income stream after her death will be inherited by her heirs. Assuming that the pure time preferences of her heirs remain the same, the value of utility at $s_{1}$ is $\mathrm{e}^{-\rho\left(s-s_{1}\right)} u(c(s))$, and the present value at $t$ of future utility $u(c(s))$ is $\beta \mathrm{e}^{-\rho\left(s_{1}-t\right)} \mathrm{e}^{-\rho\left(s-s_{1}\right)}=\beta \mathrm{e}^{-\rho(s-t)}$. Therefore, the present value at $t$ of expected future utility $u(c(s))$ is:

$$
\left(1-\Omega_{t}(s)\right) \beta \mathrm{e}^{-\rho(s-t)} u(c(s))
$$

where $\beta$ is the "altruistic" factor.

Given these two possibilities, the present value (at time $t$ ) of expected future contracted utility $u(c(s))$ at $t$ is:

$$
\left[\Omega_{t}(s) \mathrm{e}^{-\rho(s-t)}+\left(1-\Omega_{t}(s)\right) \beta \mathrm{e}^{-\rho(s-t)}\right] u(c(s)) .
$$

Hence, the discount function for $s$ to $t$ is:

$$
D(t, s)=(1-\beta) \overbrace{\mathrm{e}^{-\rho(s-t)} \mathrm{e}^{-\lambda\left(s^{\gamma}-t^{\gamma}\right)}}^{\text {short-run factor }}+\beta \overbrace{\mathrm{e}^{-\rho(s-t)}}^{\text {long-run factor }} .
$$

$D(t, s)$ can be regarded as a convex combination of a short-run exponential discount factor $\mathrm{e}^{-\rho(s-t)} \mathrm{e}^{-\lambda\left(s^{\gamma}-t^{\gamma}\right)}$ with weight $(1-\beta)$ and a long-run exponential discount factor $\mathrm{e}^{-\rho(s-t)}$ with weight $\beta .^{30}$

The discount function [Eq. (12)] satisfies the sufficient condition of dynamic preference reversal established in Proposition 2 (see the proof shown in Appendix 3). In

\footnotetext{
${ }^{28}$ Discounting behavior is the same as that considered in Halevy (2005) model if $\beta=0$, which cannot explain dynamic preference reversal and which reduces to exponential discounting if $\beta=1$. Therefore, we focus on the case of $0<\beta<1$.

${ }^{29}$ We do not consider burn-in and adult periods. For the former, the individual is still a child and hardly considers bequests made, and discounting behavior is the same as that illustrated in Halevy (2005) model; in the latter case of the adult period, the hazard rate of death is constant, and the corresponding discount function is time invariant and hyperbolic.

${ }^{30} D(t, s)$ in Eq. (12) is similar in form to the expectation of the stochastic hyperbolic discounting function proposed by Harris and Laibson (2013). However, $\pi(\tau)$ in Harris and Laibson (2013) is constant. The discount function for $\beta=0$ is reduced to Halevy (2005) model.
} 
other words, the discounting behavior characterized by Eq. (12) exhibits dynamic preference reversal over time. A close inspection of Eq. (12) shows the underlying cause of this phenomenon. The DM updates her survival probabilities over time. This updating process has two marginal effects on the expected future utility $E_{t}[u(c(s))]$ that will emerge at time $s$ : (a) it increases the probability of future utility $E_{t}[u(c(s))]$ consumed by oneself over time because as $t$ approaches $s$, the conditional survival probability $\Omega_{t}(s)$ increases; (b) it reduces the probability of the future utility consumed by one's heirs. Effect (a) is proportional for $E_{t}\left[u\left(c\left(s_{1}\right)\right)\right]$ and $E_{t}\left[u\left(c\left(s_{2}\right)\right)\right], s_{2}>s_{1} .{ }^{31}$ However, effect (b), which is negative, is larger for $E_{t}\left[u\left(c\left(s_{2}\right)\right)\right]$ than for $E_{t}\left[u\left(c\left(s_{1}\right)\right)\right]$. These combined effects cause the discount value to increase with the passage of decision time and are larger for rewards received earlier than for rewards received later. Hence, the marginal rate of substitution at $s_{1}$ for utility at $s_{2}$ decreases with the passage of decision time. This consideration leads the DM to choose the smaller, earlier reward in the near future when she is allowed to re-optimize. Our analysis is analogous to that of Dasgupta and Maskin (2005). In their model, the DM updates information received earlier with the passage of time, and the corresponding updating information process results in dynamic preference reversal. In our model, the DM updates her survival information based on the mortality risk, which reflects a real-life situation and has a concrete economic interpretation.

To use a concrete example, consider a person at the age of 70 who is comparing two monetary rewards to be received when she turns 81 and 82 years. Suppose that the later reward is large enough for her to prefer it over the earlier reward at the age of 70. After ten years, she advances to the age of 80 and reassesses her chances of being alive at 81 and 82 . She realizes that her chances of enjoying these rewards by herself are more likely now than when she was 70 . Furthermore, the increase in the conditional survival probability of reaching 81 is greater than that of reaching $82 .{ }^{32}$ On the other hand, the likelihood that she will bestow the rewards on her heirs is simultaneously reduced. As the utility of bestowing is only a fraction $(\beta)$ of that of her own consumption, the increase in utility from her own consumption has a dominant effect. Consequently, at the age of 80, the utility of her enjoying the earlier reward may well increase sufficiently due to the increase in the updated conditional survival probability of reaching the age of 81 relative to that of reaching the age of 82 , so she exhibits preference reversal and prefers the earlier reward to the later one.

We illustrate the MRS's decreasing behavior by plotting the marginal rate of substitution $\operatorname{MRS}_{t}(s, s+1)$. Without a loss of generality, we assume parameters $\beta=0.5$, $\rho=0, \lambda=0.04, \gamma=1.2$ and $s=4 .{ }^{33}$ Figure 3 shows that $\operatorname{MRS}_{t}(s, s+1)$ decreases as

\footnotetext{
31 When the decision time changes from $t_{1}$ to $t_{2}$, the expected present value of future utility $u(c(s))$ changes from $\Omega_{t_{1}}(s) u(c(s))$ to $\Omega_{t_{2}}(s) u(c(s))$. Therefore, the proportion is $\frac{\Omega_{t_{1}}\left(s_{2}\right) u\left(c\left(s_{2}\right)\right)}{\Omega_{t_{1}}\left(s_{1}\right) u\left(c\left(s_{1}\right)\right)}=\frac{\Omega\left(s_{2}\right) u\left(c\left(s_{2}\right)\right)}{\Omega\left(s_{1}\right) u\left(c\left(s_{1}\right)\right)}$.

32 At time $t$, the conditional probabilities of surviving up to $t_{s}$ and $t_{l}$ are denoted by $\Omega_{t}\left(t_{s}\right)$ and $\Omega_{t}\left(t_{l}\right)$, $t_{s}<t_{l}$, respectively. It can be shown that $\frac{\Omega_{t}\left(t_{l}\right)}{\Omega_{t}\left(t_{s}\right)}=\frac{\Omega\left(t_{l}\right)}{\Omega\left(t_{s}\right)}$ and that the latter fraction does not depend on the decision time $t$. As time $t$ increases, both survival probabilities increase. Because $\frac{\Omega_{t}\left(t_{l}\right)}{\Omega_{t}\left(t_{S}\right)}$ is constant given $t_{s}$ and $t_{l}$, the increase in $\Omega_{t}\left(t_{s}\right)$ is greater than the increase in $\Omega_{t}\left(t_{l}\right)$.

33 The life expectancy value is $E[X]=\left(\frac{1}{\lambda}\right)^{\frac{1}{\gamma}} \Gamma\left(1+\frac{1}{\gamma}\right)=\left(\frac{1}{0.04}\right)^{\frac{1}{1.2}} \Gamma\left(1+\frac{1}{1.2}\right)=13.8$. where $\Gamma(\cdot)$ is the gamma function that satisfies $\Gamma(\eta)=\int_{0}^{\infty} x^{\eta-1} e^{-x} d x$.
} 


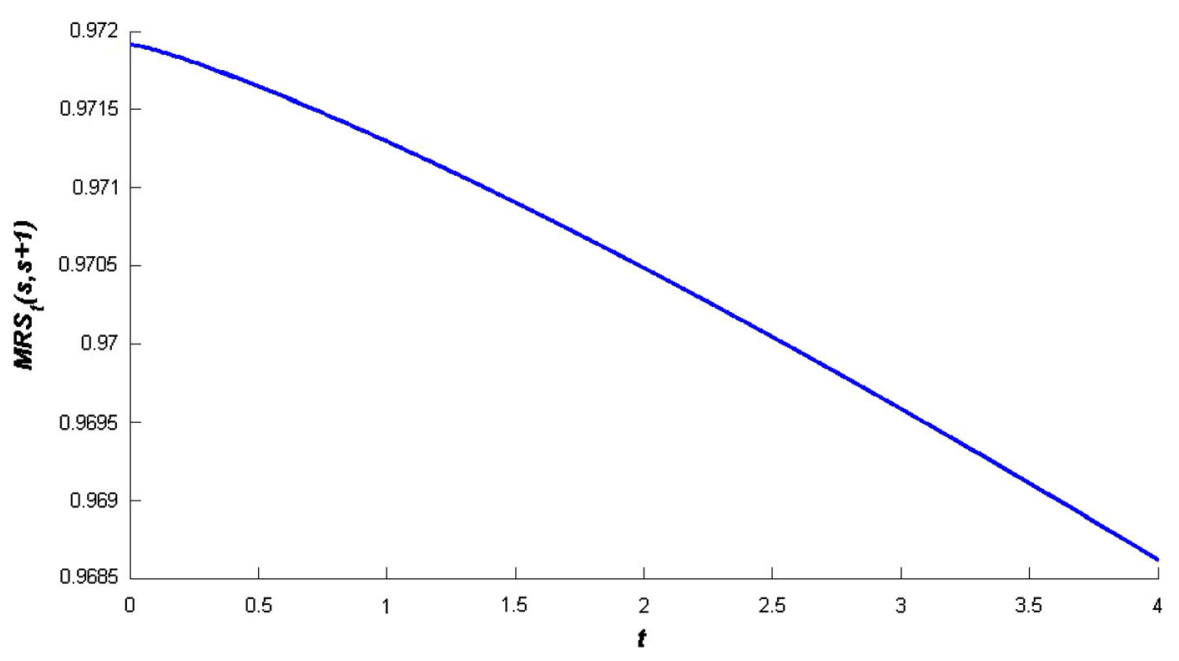

Fig. 3 The MRS of time-inconsistent behavior

the decision time $t$ approaches the earlier alternative. From Propositions 1 and 2, the DM has a time-inconsistent preference and will show dynamic preference reversal.

Researchers use hyperbolic discounting - a functional form of discounting that accommodates present bias to characterize non-stationary preferences-to explain preference reversals. We next determine whether the discount function of Eq. (12) is hyperbolic. From the definition of hyperbolic discounting used in Read (2001), Read and Roelofsma (2003), Scholten and Read (2006), Noor (2009), Kinari et al. (2009) and Halevy (2005), a discount function is hyperbolic if its marginal rate of substitution increases as the two alternatives shift equally into the future when the decision time is fixed. ${ }^{34}$ In our model, with a fixed decision time, the marginal rate of substitution first decreases and then increases, implying that the discount function of Eq. (12) is non-hyperbolic (see Appendix 4).

For a fixed decision time, the marginal rate of substitution first decreases and then increases in our discounting model as the two alternatives shift into the future. As in our previous analysis, this result is determined by the two marginal effects on the expected future utility. As the two alternatives shift equally into the future, the first effect decreases the probability of future utility $E[u(c(s))]$ being consumed by oneself because of reduced remaining lifetime as one ages; the second effect increases the probability of future utility being consumed by one's heirs. ${ }^{35}$ The first effect, which negatively affects the expected future utility, is greater for the later alternative than for the earlier one due to the increasing hazard rate of death that causes the marginal rate of substitution to decrease; by contrast, the second effect is greater for the earlier alternative than for the later one, causing the marginal rate of substitution to increase. When the two timed alternatives will emerge in the near future, the probability that the utility is inherited and consumed by heirs is very small. Consequently, the first

\footnotetext{
34 Without a loss of generality, we assume that the decision time is set at 0 .

35 Here, the decision time is set at 0 and $E=E_{0}$.
} 


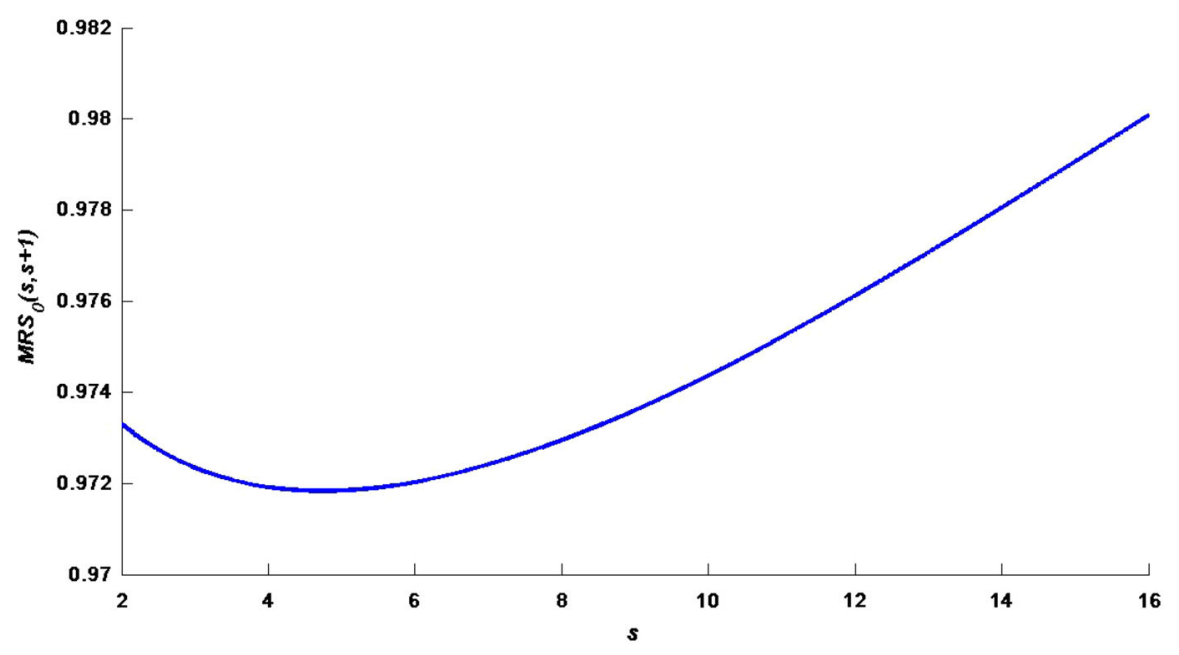

Fig. 4 The MRS of non-stationary behavior

effect dominates the second, which leads the marginal rate of substitution to decline when shifting the two alternatives into the future. By contrast, when the two timed alternatives are set in the far distant future, the probability of utility being consumed by the DM is very low because of an individual's limited lifetime. As a result, the second effect dominates the first, causing the marginal rate of substitution to increase. Our analysis shows that the DM's non-constant hazard rate of death contributes to the formation of non-stationary preferences and that the DM's non-stationary preferences are characterized by non-hyperbolic discounting behavior.

We illustrate the non-hyperbolic discounting behavior of Eq. (12) by plotting the marginal rate of substitution $\operatorname{MRS}_{t}(s, s+1)$. As in Fig. 3, we adopt the

parameters $\beta=0.5, \rho=0, \lambda=0.04$, and $\gamma=1.2$ and assume that the decision time is present, i.e., $t=0$. Figure 4 shows that $\operatorname{MRS}_{0}(s, s+1)$ first decreases and then increases as the two timed alternatives at $s$ and $s+1$ shift equally into the future. This pattern of the marginal rate of substitution means that the corresponding discounting behavior is non-hyperbolic.

From the above analysis, we make the following proposition:

Proposition 3 If the DM cares about her bequest and distinguishes between it and her own consumption, then discounting behavior is non-hyperbolic, and the process of updating survival information accounts for dynamic preference reversal behavior.

As shown above, the process of updating survival information accounts for dynamic preference reversal behavior. We next show that the marginal rate of substitution for our discount function [Eq. (12)] decreases with calendar time $t$ (see the proof shown in Appendix 5), which implies that the DM's preferences are time varying. As the DM approaches the end of her life with a non-constant hazard rate of death, she updates her survival information and recognizes changing survival probabilities. As the utility derived from a bequest is a fraction $\beta$ of that derived from her own consumption, the discount function is time varying as she ages. 


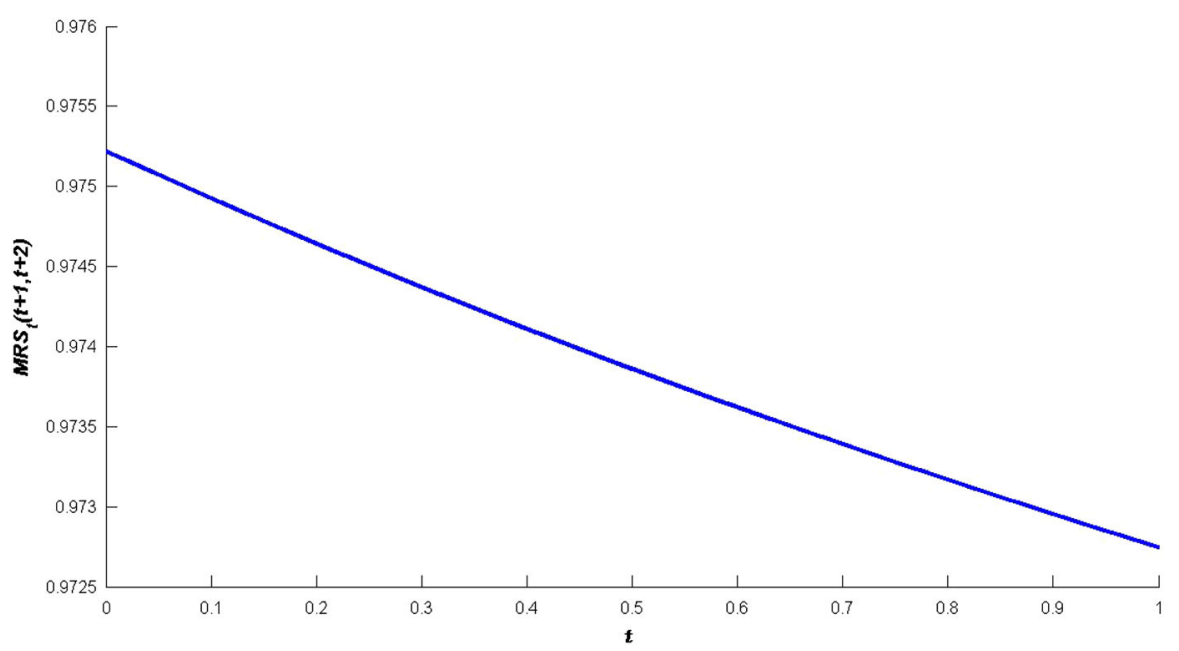

Fig. 5 The MRS of time-varying behavior

We illustrate time-varying behavior by plotting a marginal rate of substitution $\operatorname{MRS}_{t}\left(t+\Delta_{1}, t+\Delta_{2}\right)$. In the graph, we use the parameters $\beta=0.5, \rho=0, \lambda=$ 0.04, $\gamma=1.2, \Delta_{1}=1$ and $\Delta_{2}=2$. Figure 5 shows that $\operatorname{MRS}_{t}\left(t+\Delta_{1}, t+\Delta_{2}\right)$ decreases as calendar time $t$ increases.

Our analysis demonstrates that the DM's preferences derived from our discounting model are non-stationary, time varying and time inconsistent. The DM's non-constant hazard rate of death leads to the non-stationarity of her preferences. We also show that the preferences from our model are non-hyperbolic. The process of updating survival information is the underlying driver that renders them time varying and time inconsistent. Therefore, survival information updating may be another underlying driver of dynamic preference reversal behavior. Our results and those of Dasgupta and Maskin (2005) serve as a theoretical foundation that explains the experimental results of Halevy (2005). ${ }^{36}$

\section{Conclusion}

In this study, we analyze the underlying causes of dynamic preference reversal. We first characterize the three inter-related notions of time invariance, stationarity and time consistency from the marginal rate of substitution and establish sufficient conditions that lead to dynamic preference reversal. We consider mortality risk and an altruistic factor in our discounting model and present a discount function that is nonstationary, time varying and non-hyperbolic. The DM's preference is non-stationary because of the non-constant hazard rate of death. Moreover, the process of updating

\footnotetext{
36 Notably, our results differ from those of Azfar (1999) and Halevy (2005). In the model developed by Azfar (1999), even when the DM updates her survival information, the DM's choice is time consistent. However, when the DM updates her survival information, the DM exhibits dynamic preference reversals in our model..
} 
survival information renders discounting behavior time varying. The DM becomes more inclined to receive the smaller but earlier reward with the passage of the decision time. Our analysis shows that the process of updating uncertain information may account for the occurrence of dynamic preference reversal.

Acknowledgements This study is supported by the National Natural Science Foundations of China (Grant nos. 71501065, 71221001, 71790593, 71572055, 71573077 and 71850012).

Open Access This article is distributed under the terms of the Creative Commons Attribution 4.0 International License (http://creativecommons.org/licenses/by/4.0/), which permits unrestricted use, distribution, and reproduction in any medium, provided you give appropriate credit to the original author(s) and the source, provide a link to the Creative Commons license, and indicate if changes were made.

\section{Appendix 1}

Halevy (2015) shows that preferences $\left\{\succsim_{t}\right\}_{t=0}^{\infty}$ are time invariant if for every $t, t^{\prime}>0$, $\xi>0, \psi>0$ and $\Delta_{1}, \Delta_{2}>0$ :

$$
\left(\xi, t+\Delta_{1}\right) \sim_{t}\left(\psi, t+\Delta_{2}\right) \Leftrightarrow\left(\xi, t^{\prime}+\Delta_{1}\right) \sim_{t^{\prime}}\left(\psi, t^{\prime}+\Delta_{2}\right)
$$

From the definition of discounted utility, i.e., Equation (1), and the definition of a time-invariant preference, i.e., Eq. (13), the necessary and sufficient condition for time-invariant preferences is

$$
D\left(t, t+\Delta_{1}\right) u(\xi)=D\left(t, t+\Delta_{2}\right) u(\psi) \Leftrightarrow D\left(t^{\prime}, t^{\prime}+\Delta_{1}\right) u(\xi)=D\left(t^{\prime}, t^{\prime}+\Delta_{2}\right) u(\psi),
$$

and therefore

$\operatorname{MRS}_{t}\left(t+\Delta_{1}, t+\Delta_{2}\right)=\frac{D\left(t, t+\Delta_{2}\right)}{D\left(t, t+\Delta_{1}\right)}=\frac{D\left(t^{\prime}, t^{\prime}+\Delta_{2}\right)}{D\left(t^{\prime}, t^{\prime}+\Delta_{1}\right)}=\operatorname{MRS}_{t^{\prime}}\left(t^{\prime}+\Delta_{1}, t^{\prime}+\Delta_{2}\right)$.

Therefore, we reach the first conclusion for Proposition 1. From similar deductions, we can draw the second and third conclusions for Proposition 1

\section{Appendix 2}

Dynamic preference reversal means that for all $\sigma>0$,

$$
\left(\xi, t^{\prime}+\Delta_{1}\right) \sim_{s}\left(\psi, t^{\prime}+\Delta_{2}\right) \Rightarrow\left(\xi, t^{\prime}+\Delta_{1}\right) \succ_{s+\sigma}\left(\psi, t^{\prime}+\Delta_{2}\right)
$$

and

$$
\left(\xi, t^{\prime}+\Delta_{1}\right) \sim_{s}\left(\psi, t^{\prime}+\Delta_{2}\right) \Rightarrow\left(\xi, t^{\prime}+\Delta_{1}\right) \prec_{s-\sigma}\left(\psi, t^{\prime}+\Delta_{2}\right)
$$

where $t^{\prime}+\Delta_{1}>s+\sigma$. 
From Eqs. (14)-(15) and the definition of discounted utility, i.e., Equation (1), we have

$$
\begin{array}{r}
D\left(s, t^{\prime}+\Delta_{1}\right) u(\xi)=D\left(s, t^{\prime}+\Delta_{2}\right) u(\psi) \\
\Rightarrow D\left(s+\sigma, t^{\prime}+\Delta_{1}\right) u(\xi)>D\left(s+\sigma, t^{\prime}+\Delta_{2}\right) u(\psi),
\end{array}
$$

and

$$
\Rightarrow D\left(s-\sigma, t^{\prime}+\Delta_{1}\right) u(\xi)<D\left(s-\sigma, t^{\prime}+\Delta_{2}\right) u(\psi)
$$

From Eqs. (16)-(17), we have $\mathrm{MRS}_{s-\sigma}\left(t^{\prime}+\Delta_{1}, t^{\prime}+\Delta_{2}\right)>\mathrm{MRS}_{s}$ $\left(t^{\prime}+\Delta_{1}, t^{\prime}+\Delta_{2}\right)>\operatorname{MRS}_{s+\sigma}\left(t^{\prime}+\Delta_{1}, t^{\prime}+\Delta_{2}\right)$ for all $\sigma>0$. Therefore, $\operatorname{MRS}_{s+\sigma}\left(t^{\prime}+\right.$ $\left.\Delta_{1}, t^{\prime}+\Delta_{2}\right)$ decreases as $\sigma$ increases. Hence, the sufficient condition for dynamic preference reversal is that the marginal rate of substitution $\operatorname{MRS}_{s+\sigma}\left(t^{\prime}+\Delta_{1}, t^{\prime}+\Delta_{2}\right)$ decreases as $\sigma$ increases.

Define $t=s+\sigma$. From the above analysis, the sufficient condition for dynamic preference reversal is that the marginal rate of substitution $\operatorname{MRS}_{t}\left(t^{\prime}+\Delta_{1}, t^{\prime}+\Delta_{2}\right)$ decreases as the decision time $t$ shifts into the future.

\section{Appendix 3}

From the expression of the discount function Eq. (12), the marginal rate of the substitution of utility $\operatorname{MRS}_{t}\left(t^{\prime}+\Delta_{1}, t^{\prime}+\Delta_{2}\right)$ satisfies:

$$
\operatorname{MRS}_{t}\left(t^{\prime}+\Delta_{1}, t^{\prime}+\Delta_{2}\right)=\mathrm{e}^{-\rho\left(\Delta_{2}-\Delta_{1}\right)} \frac{(1-\beta) \mathrm{e}^{-\lambda\left(\left(t^{\prime}+\Delta_{2}\right)^{\gamma}-t^{\gamma}\right)}+\beta}{(1-\beta) \mathrm{e}^{-\lambda\left(\left(t^{\prime}+\Delta_{1}\right)^{\gamma}-t^{\gamma}\right)}+\beta}
$$

Thus,

$$
\frac{\mathrm{dMRS}_{t}\left(t^{\prime}+\Delta_{1}, t^{\prime}+\Delta_{2}\right)}{\mathrm{d} t}=\mathrm{e}^{-\rho\left(\Delta_{2}-\Delta_{1}\right)} \frac{\beta(1-\beta) \lambda \gamma t^{\gamma-1} \mathrm{e}^{-\lambda t^{\gamma}}\left[\mathrm{e}^{-\lambda\left(t^{\prime}+\Delta_{2}\right)^{\gamma}}-\mathrm{e}^{-\lambda\left(t^{\prime}+\Delta_{1}\right)^{\gamma}}\right]}{\left((1-\beta) \mathrm{e}^{-\lambda\left(\left(t^{\prime}+\Delta_{1}\right)^{\gamma}-t^{\gamma}\right)}+\beta\right)^{2}}<0,
$$

which means that the discount function Eq. (12) satisfies the condition for dynamic preference reversal. Therefore, the discount function Eq. (12) can explain the occurrence of dynamic preference reversal.

\section{Appendix 4}

According to Read (2001), Read and Roelofsma (2003), Scholten and Read (2006), Noor (2009), Kinari, et al. (2009) and Halevy (2005), with the fixed decision time, if the marginal rate of substitution increases as the two alternatives shift equally into the future, this discounting behavior is hyperbolic. 
Without a loss of generality, we assume that the decision time is set to 0 . From the definition of the marginal rate of substitution $\operatorname{MRS}_{0}(s, s+\tau)$ and Eq. (12), we have:

$$
\operatorname{MRS}_{0}(s, s+\tau)=\mathrm{e}^{-\rho \tau} \frac{(1-\beta) \mathrm{e}^{-\lambda(s+\tau)^{\gamma}}+\beta}{(1-\beta) \mathrm{e}^{-\lambda s^{\gamma}}+\beta} .
$$

Therefore,

$$
\frac{d \operatorname{MRS}_{0}(s, s+\tau)}{\mathrm{d} s}=\mathrm{e}^{-\rho \tau} \frac{g(s)}{\left((1-\beta) \mathrm{e}^{-\lambda s^{\gamma}}+\beta\right)^{2}},
$$

where

$$
\begin{array}{r}
g(s)=(1-\beta) \lambda \gamma\left\{(1-\beta) \mathrm{e}^{-\lambda(s+\tau)^{\gamma}-\lambda s^{\gamma}}\left[s^{\gamma-1}-(s+\tau)^{\gamma-1}\right]\right. \\
\left.+\beta\left[s^{\gamma-1} \mathrm{e}^{-\lambda s^{\gamma}}-(s+\tau)^{\gamma-1} \mathrm{e}^{-\lambda(s+\tau)^{\gamma}}\right]\right\} .
\end{array}
$$

From Eq. (18), we can see that $(1-\beta) \mathrm{e}^{-\lambda(s+\tau)^{\gamma}-\lambda s^{\gamma}}\left[s^{\gamma-1}-(s+\tau)^{\gamma-1}\right]$ is less than zero; $\beta\left[s^{\gamma-1} \mathrm{e}^{-\lambda s^{\gamma}}-(s+\tau)^{\gamma-1} \mathrm{e}^{-\lambda(s+\tau)^{\gamma}}\right]$ is small but greater than zero when $s$ is sufficiently large. As $s$ increases, $g(s)$ is initially less than zero and then eventually becomes greater than zero. Therefore, from Eq. (18), as time $s$ increases, the marginal substitution $\operatorname{MRS}_{0}(s, s+\tau)$ first decreases and then increases. Hence, from the definition of hyperbolic discounting, Eq. (12) is not a hyperbolic discount function since $\operatorname{MRS}_{0}(s, s+\tau)$ does not monotonically increase with $s$.

\section{Appendix 5}

From the definition of the marginal rate of substitution $\operatorname{MRS}_{t}\left(t+\Delta_{1}, t+\Delta_{2}\right)$ and Eq. (12), we have:

$$
\operatorname{MRS}_{t}\left(t+\Delta_{1}, t+\Delta_{2}\right)=\mathrm{e}^{-\rho\left(\Delta_{2}-\Delta_{1}\right)} \frac{(1-\beta) \mathrm{e}^{-\lambda\left(\left(t+\Delta_{2}\right)^{\gamma}-t^{\gamma}\right)}+\beta}{(1-\beta) \mathrm{e}^{-\lambda\left(\left(t+\Delta_{1}\right)^{\gamma}-t^{\gamma}\right)}+\beta} .
$$

Therefore,

$$
\frac{d \mathrm{MRS}_{t}\left(t+\Delta_{1}, t+\Delta_{2}\right)}{d t}=\frac{\lambda(1-\beta) \mathrm{e}^{-\lambda t^{\gamma}} \mathrm{e}^{-\rho\left(\Delta_{2}-\Delta_{1}\right)}\left[h_{1}(t)+h_{2}(t)+h_{3}(t)\right]}{\left((1-\beta) \mathrm{e}^{-\lambda\left(\left(t+\Delta_{1}\right)^{\gamma}-t^{\gamma}\right)}+\beta\right)^{2}},
$$

where

$$
\begin{gathered}
h_{1}(t)=\beta t^{\gamma-1}\left[\mathrm{e}^{-\lambda\left(t+\Delta_{2}\right)^{\gamma}}-\mathrm{e}^{-\lambda\left(t+\Delta_{1}\right)^{\gamma}}\right]<0, \\
h_{2}(t)=\beta\left[\mathrm{e}^{-\lambda\left(t+\Delta_{1}\right)^{\gamma}}\left(t+\Delta_{1}\right)^{\gamma-1}-\mathrm{e}^{-\lambda\left(t+\Delta_{2}\right)^{\gamma}}\left(t+\Delta_{2}\right)^{\gamma-1}\right]<0,
\end{gathered}
$$


$h_{3}(t)=(1-\beta) \mathrm{e}^{-\lambda\left(t+\Delta_{1}\right)^{\gamma}} \mathrm{e}^{-\lambda\left(t+\Delta_{2}\right)^{\gamma}}\left[\mathrm{e}^{-\lambda\left(t+\Delta_{1}\right)^{\gamma}}\left(t+\Delta_{1}\right)^{\gamma-1}-\mathrm{e}^{-\lambda\left(t+\Delta_{2}\right)^{\gamma}}\left(t+\Delta_{2}\right)^{\gamma-1}\right]<0$,

for $\Delta_{2}>\Delta_{1}>0$ and $\gamma>1$.

Therefore, the marginal rate of substitution $\operatorname{MRS}_{t}\left(t+\Delta_{1}, t+\Delta_{2}\right)$ decreases as the calendar time $t$ increases.

\section{References}

Attema, A. E., Bleichrodt, H., Rohde, K. I. M., \& Wakker, P. P. (2010). Time-tradeoff sequences for analyzing discounting and time inconsistency. Management Science, 56(11), 2015-2030.

Azfar, O. (1999). Rationalizing hyperbolic discounting. Journal of Economic Behavior \& Organization, 38(2), 245-252.

Chen, S., Li, Z., \& Zeng, Y. (2014). Optimal dividend strategies with time-inconsistent preferences. Journal of Economic Dynamics and Control, 46, 150-172.

Dasgupta, P., \& Maskin, E. (2005). Uncertainty and hyperbolic discounting. The American Economic Review, 95(4), 1290-1299.

De Nardi, M. (2004). 'Wealth inequality and intergenerational links'. Review of Economic Studies, 71(3), $743-768$.

Ekeland, I., \& Pirvu, T. A. (2008). Investment and consumption without commitment. Mathematics and Financial Economics, 2(1), 57-86.

Fishburn, P. C., \& Rubinstein, A. (1982). Time preference. International Economic Review, 23(3), 677-694.

Frederick, S., Loewenstein, G. F., \& O’Donoghue, T. (2002). Time discounting and time preference: a critical review. Journal of Economic Literature, 40(2), 351-401.

Grenadier, S. R., \& Wang, N. (2007). Investment under uncertainty and time-inconsistent preferences. Journal of Financial Economics, 84(1), 2-39.

Gul, F., \& Pesendorfer, W. (2001). Temptation and self-control. Econometrica, 69(6), 1403-1435.

Halevy, Y. (2005). Diminishing impatience: Disentangling time preference from uncertain lifetime. Discussion Paper 05-15, UBC Department of Economics, [336, 348, 350].

Halevy, Y. (2015). Time consistency: stationarity and time invariance. Econometrica, 83(1), 335-352.

Harris, C., \& Laibson, D. (2013). Instantaneous gratification. The Quarterly Journal of Economics, 128(1), 205-248.

Harvey, C.M. (1986). Value functions for infinite-period planning. Management Science, 32(9), 1123-1139.

Hoch, S. J., \& Loewenstein, G. F. (1991). Time-inconsistent preferences and consumer self-control. Journal of Consumer Research, 17(4), 492-507.

Keren, G., \& Roelofsma, P. (1995). Immediacy and certainty in intertemporal choice. Organizational Behavior and Human Decision Making, 63(3), 287-297.

Kinari, Y., Ohtake, F., \& Tsutsui, Y. (2009). Time discounting: declining impatience and interval effect. Journal of Risk and Uncertainty, 39(1), 87-112.

Kotlikoff, L. J., \& Summers, L. H. (1981). The role of intergenerational transfers in aggregate capital accumulation. Journal of Political Economy, 89(4), 706-732.

Kuehlwein, M. (1993). Life-cycle and altruistic theories of saving with lifetime uncertainty. Review of Economics and Statistics, 75(1), 38-47.

Loewenstein, G. F., \& Prelec, D. (1992). Anomalies in intertemporal choice: Evidence and an interpretation. The Quarterly Journal of Economics, 107(2), 573-597.

Mazur, J.E. (1987). An adjusting procedure for studying delayed reinforcement. In M. L. Commons, J. E. Mazur, J. A. Nevin, \& H. Rachlin (Eds.), Quantitative analyses of behavior, Vol. 5. The effect of delay and of intervening events on reinforcement value (pp. 55-73). Hillsdale, NJ, US: Lawrence Erlbaum Associates, Inc.

Miao, J. (2008). Option exercise with temptation. Economic Theory, 34(3), 473-501.

Noor, J. (2009). Hyperbolic discounting and the standard model: Eliciting discount functions. Journal of Economic Theory, 144(5), 2077-2083.

Noor, J. (2011). Intertemporal choice and the magnitude effect. Games and Economic Behavior, 71, 255-270.

O’Donoghue, T., \& Rabin, M. (1999). Doing it now or later. The American Economic Review, 89(1), $103-124$. 
O’Donoghue, T., \& Rabin, M. (2015). Present bias: Lessons learned and to be learned. The American Economic Review, 105(5), 273-279.

Pan, J., Webb, C. S., \& Zank, H. (2015). An extension of quasi-hyperbolic discounting to continuous time. Games and Economic Behavior, 89, 43-55.

Pennesi, D. (2017). Uncertain discount and hyperbolic preferences. Theory and Decision, 83(3), 315-336.

Phelps, E. S., \& Pollak, R. A. (1968). 'On second-best national saving and game-equilibrium growth. The Review of Economic Studies, 35(2), 185-199.

Read, D. (2001). Is time-discounting hyperbolic or subadditive? Journal of Risk and Uncertainty, 23(1), 5-32.

Read, D. (2004). Intertemporal choice. In D. Koehler \& N. Harvey (Eds.), The Blackwell handbook of judgment and decision making. Oxford: Blackwell.

Read, D., \& Roelofsma, P. H. (2003). Subadditive versus hyperbolic discounting: A comparison of choice and matching. Organizational Behavior and Human Decision Processes, 91(2), 140-153.

Sayman, S., \& Öncüler, A. (2009). An investigation of time inconsistency. Management Science, 55(3), $470-482$.

Scholten, M., \& Read, D. (2006). Discounting by intervals: A generalized model of intertemporal choice. Management Science, 52(9), 1424-1436.

Sozou, P. D. (1998). On hyperbolic discounting and uncertain hazard rates. Proceedings of the Royal Society of London, 265(1409), 2015-2020.

Strotz, R. H. (1955). Myopia and inconsistency in dynamic utility maximization. The Review of Economic Studies, 23(3), 165-180.

Tversky, A., \& Kahneman, D. (1991). Loss aversion in riskless choice: A reference-dependent model. Quarterly Journal of Economics, 106, 1039-1061.

Yaari, M. E. (1965). Uncertain lifetime, life insurance, and the theory of the consumer. The Review of Economic Studies, 32(2), 137-150.

Zou, Z., Chen, S., \& Wedge, L. (2014). Finite horizon consumption and portfolio decisions with stochastic hyperbolic discounting. Journal of Mathematical Economics, 52(10), 70-80.

Publisher's Note Springer Nature remains neutral with regard to jurisdictional claims in published maps and institutional affiliations. 